\title{
TellStory-Analyzer: Um mecanismo para aprimorar a externalização do conhecimento
}

\author{
Miguel G. P. Carvalho, Ruben P. Albuquerque, \\ Marcos R. S. Borges, Vanessa Braganholo
}

Programa de Pós Graduação em Informática - Universidade Federal do Rio de Janeiro (UFRJ) - Rio de Janeiro, RJ, Brasil

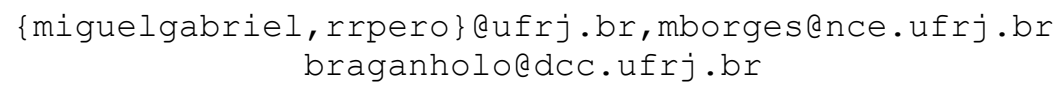

\begin{abstract}
People and organizations have a need to review past events with the intention of reconstructing knowledge for several purposes. Group Storytelling is an important approach to knowledge retrieval and externalization through the collaborative construction of stories. However, in a Group Storytelling dynamic, past experiences show that it is not always easy to get the group committed. This paper aims to demonstrate that through computational mechanisms it is possible to increase the participation and contribution of the group. For this we've used the TellStory tool, that implements the Group Storytelling approach, and the TellStory-Analyzer mechanism, that aims to make a quantitative and qualitative analysis of the stories and also to analyze the participation and contribution of those involved.
\end{abstract}

Resumo. Pessoas e organizações têm necessidade de recordar eventos e acontecimentos passados com o intuito de reconstruir o conhecimento para diversas finalidades. O Group Storytelling é uma importante técnica de recuperação de conhecimento, através de composição de narrativas em grupo visando auxiliar na externalização. No entanto, experiências anteriores mostram que nem sempre é fácil obter o comprometimento do grupo na dinâmica do Group Storytelling. Este artigo visa a demonstrar que através de mecanismos computacionais é possível aumentar a participação e contribuição do grupo. Para isso foi utilizado a ferramenta TellStory que implementa o Group Storytelling e o mecanismo TellStory-Analyzer que tem por objetivo fazer uma análise quantitativa e qualitativa das histórias e também analisar a participação e contribuição dos envolvidos.

\section{Introdução}

O conhecimento é o ativo mais valioso de uma organização [Prusak, 2001]. A gestão adequada deste bem pode estabelecer diferenciais competitivos para empresas [Alavi e Leidner, 1999]. Pessoas e organizações têm necessidade de recordar eventos e acontecimentos passados com intuito de reconstrução do conhecimento para diversas finalidades, como, por exemplo, a tomada de decisões futuras, registro de uma memória organizacional e melhor entendimento de um acontecimento passado.

O conhecimento pode ser caracterizado de duas formas: conhecimento tácito e explícito [Nonaka, 1991]. O conhecimento tácito é pessoal, adquirido através da experiência e permanece no intelecto individual. Já o conhecimento explícito envolve a captura e representação dos fatos, sendo facilmente codificado e transmitido [Paula e 
Cianconi, 2007]. A dificuldade no processo de gestão do conhecimento está em como permitir a sua externalização - transformação do conhecimento tácito para explícito - de uma forma eficaz.

Uma técnica utilizada para a externalização do conhecimento é a técnica de Group Storytelling, ou contagem de histórias em grupo, onde pessoas contribuem síncrona ou assincronamente para a reconstrução de eventos e fatos através da contagem de histórias. Os participantes do Group Storytelling podem estar no mesmo local ou geograficamente distribuídos [Valle, Prinz e Borges, 2002]. A contagem de histórias é um caminho natural para a transformação de conhecimento tácito em explícito [Carminatti, Borges e Gomes, 2005].

Para apoiar a construção colaborativa de histórias utilizando a técnica de Group Storytelling, Perret, Borges e Santoro (2004) propuseram a ferramenta TellStory ${ }^{1}$. O TellStory é um importante mecanismo de recuperação do conhecimento, testado em diversos contextos [Perret, 2004; Carminatti, 2006]. Contudo, a ferramenta possui um problema recorrente em aplicações práticas, que é a falta de comprometimento dos participantes durante a dinâmica, gerando poucos fragmentos e uma história pobre [Laporti, Borges e Braganholo, 2009]. O TellStory-Analyzer, proposto neste trabalho, tem por objetivo estimular a maior cooperação e participação das pessoas envolvidas na contagem das histórias, a fim de promover uma maior externalização do conhecimento. A idéia é fornecer recursos para o gerenciamento global das histórias com apoio de análises qualitativas e quantitativas dos fragmentos, de forma que os participantes sejam estimulados a participar dos relatos.

A partir de um experimento realizado com dois grupos utilizando a ferramenta TellStory, foram levantados requisitos para o desenvolvimento do mecanismo proposto nesse artigo - o TellStory-Analiser. Em ambos os grupos, cada participante assistiu apenas a algumas cenas de um filme, com o intuito de tentar reconstruí-lo. Adicionalmente, no segundo experimento foi proposta uma etapa para refinar a reconstrução do conhecimento. Para validação desse mecanismo foi utilizado um terceiro grupo, que foi submetido ao mesmo experimento, porém contando com o auxilio computacional do TellStory-Analyzer. Com isso, foi possível identificar evidências de que mecanismos de gerência de histórias permitem um ganho na externalização do conhecimento, pois no terceiro grupo foi possível constatar um acréscimo de qualidade e quantidade dos fragmentos e comentários descritos no TellStory.

Este artigo está organizado da seguinte maneira. A Seção 2 descreve a técnica Group Storytelling e a ferramenta TellStory. A Seção 3 descreve os dois experimentos que permitiram o levantamento de requisitos para a construção do TellStory-Analyzer e apresenta uma análise desses experimentos. Na Seção 4 apresentamos o mecanismo TellStory-Analyzer. Por fim, na Seção 5 descrevemos o terceiro experimento e o ganho com a utilização do TellStory-Analyzer, seguido da conclusão desse artigo na Seção 6.

\section{Group Storytelling}

Perret (2004) descreve três maneiras para reconstruir a história de um trabalho executado e concluído por um grupo. A primeira delas é delegar a tarefa a um dos

\footnotetext{
${ }^{1}$ http://chord.nce.ufrj.br:8080/tellstoryinv
} 
integrantes da equipe, que deverá contar todos os fatos de que se lembra. Este método é inadequado porque um único integrante não detém o conhecimento total do fato. $\mathrm{O}$ segundo método consiste em fazer entrevistas com todos os membros do grupo. Embora esse método possa permitir um enriquecimento da história em comparação com o primeiro, as entrevistas individuais ainda não estimulam a interação da equipe. $O$ terceiro método é o Group Storytelling, onde todos os membros do grupo têm a possibilidade de dialogar entre si formando no final um conhecimento que será a combinação do conhecimento de cada participante.

Valle, Prinz e Borges (2002) definem a técnica de Group Storytelling como sendo a construção de histórias na qual "mais de uma pessoa contribui, síncrona ou assincronamente, localmente ou de maneira distribuída, em vários pontos do processo, através de diversas mídias". A técnica de Group Storytelling envolve a interação entre os participantes durante um tempo pré-determinado. Ao final da aplicação dessa técnica é formada uma história, que é a combinação dos conhecimentos de todos os membros. A vantagem dessa dinâmica é que durante sua aplicação os membros do grupo têm a possibilidade de lembrar e relatar coisas não descritas inicialmente, lembranças estas que são estimuladas pela leitura do relato de outros participantes.

Como forma de auxiliar a aplicação da técnica do Group Storytelling, existem vários mecanismos e ferramentas que fornecem apoio computacional. Podem ser citados como ferramentas e mecanismo que implementam o Group Storytelling:

StoryTellingADR: sistema construído pela Universidade Técnica de Lisboa que utiliza as técnicas de Group Storytelling no contexto judicial visando à resolução de conflitos, dentro da esfera legal através da contagem de história [Silva, 2006].

BPM Story: aplicação web desenvolvida para desenho de processos através da contagem de histórias [Costa, Araújo e Borges, 2009].

Athena: aplicação web para elicitação de requisitos de um sistema. A dinâmica de elicitação de requisitos utiliza Group Storytelling [Laporti, Braganholo e Borges, 2009].

TellStory: aplicação Web que tem por objetivo a construção colaborativa de histórias, para assim explicitar o conhecimento compartilhado por um grupo [Perret, 2004].

No TellStory, a história é uma seqüência de eventos que estão ligados uns aos outros por uma seqüência de fragmentos e comentários descritos pelos membros do grupo de forma síncrona ou assíncrona. Fragmentos são pequenos trechos da história contada pelos usuários, e comentários são interações textuais dos participantes feitas a partir da leitura dos fragmentos. Entre os recursos disponibilizados por essa ferramenta estão a atribuição de papéis aos seus usuários e a organização da seqüência de eventos. O objetivo final dessa ferramenta é a construção de histórias (fatos) através de narrativas.

Para uma melhor externalização do conhecimento, seja através do Group Storytelling, seja por outra técnica, é de fundamental importância que os participantes estejam motivados e tenham comprometimento com o trabalho que está sendo desenvolvido. O mecanismo TellStory-Analyzer procura atuar nessa lacuna, permitindo um auxílio computacional para um maior comprometimento e motivação dos indivíduos através da gerência de histórias. 


\section{Experimento para Levantamento de Requisitos}

Para a elicitação dos requisitos que compõem o TellStory-Analyzer e para comprovar que mecanismos de gerência de histórias permitem uma maior externalização do conhecimento, foram feitos dois experimentos utilizando a ferramenta TellStory. Para esse estudo foram utilizados questionários e análise dos perfis e da participação dos membros. A partir desses experimentos realizou-se um estudo sobre como obter uma participação mais efetiva dos membros das histórias. história:

Nesses experimentos foram atribuídos os seguintes papéis aos membros da

- Facilitador - membro da história que tem a função de coordenar e organizar a história.

- Relator - membro que tem como função redigir a história final a partir dos fragmentos e comentários lidos.

- Usuário comum - papel desempenhado por todos os membros do grupo que constroem a história.

Ambos os experimentos foram realizados utilizando-se um filme, do qual cada participante poderia assistir apenas a alguns trechos. $\mathrm{O}$ objetivo final era recriar a história do filme juntamente com os demais participantes. Os experimentos ocorreram em três etapas:

$1^{o}$ Etapa: Os participantes assistiram aos trechos do filme atribuídos a eles.

$2^{\circ}$ Etapa: Os participantes descreveram os trechos assistidos usando o mecanismo TellStory e contribuíram para a reconstrução da história através da interação com os outros participantes. A interação com os outros participantes ocorreu pela leitura dos fragmentos dos demais participantes, pela adição de novos fragmentos e também pela inserção de comentários sobre os fragmentos já inseridos.

$3^{\circ}$ Etapa: $\mathrm{O}$ relator da história, que também podia ser um participante, fez uma síntese de todos os fragmentos e tentou reconstruir a história.

\subsection{Primeiro Experimento}

Para o primeiro experimento, foram selecionados 7 participantes, sendo que 6 assistiram a trechos do filme e relataram os fragmentos e 1 teve a função de relator. $O$ perfil dos participantes era o seguinte: 2 professores (49 e 48 anos), 1 jornalista (25 anos), 1 estudante (22 anos), 1 defensor público ( 37 anos), 1 analista de sistemas (55 anos) e 1 administrador (53 anos), que exerceu os papéis de relator e facilitador. O filme foi dividido de tal forma que os participantes precisassem uns dos outros para reconstruir a história. Nesse primeiro experimento, nenhuma parte do filme foi ocultada dos participantes. Porém, nem todos viram a mesma proporção do filme. Para os participantes de 1 a 6 , foram exibidos respectivamente $26 \%, 18 \%, 15 \%, 17 \%, 20 \%$ e $18 \%$ do filme. É importante ressaltar que havia interseção entre os trechos assistidos por alguns dos participantes.

Para a exibição do filme foi reservado uma semana. Logo depois dessa etapa houve o período de interação entre os participantes utilizando a ferramenta TellStory. Para essa segunda etapa foi também reservado uma semana. Por fim, foi utilizado o relator, que tentou reconstruir a história a partir dos fragmentos lidos. 


\subsection{Segundo Experimento}

O segundo experimento foi realizado com um grupo de 8 participantes, dos quais 6 assistiram ao filme e relataram os fragmentos e 2 extraíram a história de acordo com os fragmentos registrados. O perfil dos participantes era o seguinte: 1 músico (18 anos), 3 aposentados (61, 77 e 80 anos), 2 professores (49 e 50 anos), e 2 estudantes ( 24 e 31 anos). Os estudantes atuaram como relatores e facilitadores. Assim como no primeiro experimento, o filme foi divido de tal forma que os participantes não pudessem entender o filme sem a interação com os demais participantes. O percentual do filme assistido pelos participantes (de 1 a 6) foi: 15\%, 21\%, 16\%, 17\%, 21\% e 16\%. Novamente, alguns participantes viram trechos iguais do filme. Porém, nesse experimento ocultamos uma parte do filme de todos os participantes.

Assim como no primeiro experimento, foi reservada uma semana para a exibição do filme e mais uma semana para a interação dos participantes através do mecanismo TellStory. Porém, nesse segundo experimento foram utilizados 2 relatores para extração da história, permitindo aos demais participantes um período de votação de 2 dias para a escolha da melhor história.

\subsection{Análise dos Experimentos Iniciais}

$\mathrm{Na}$ execução dos dois experimentos foram feitas análises e estudos sobre a participação e interação dos participantes. Alguns pontos observados e levantados através de um questionário foram:

- O número de linhas escritas nos relatos não é proporcional à porcentagem do filme assistida pelos participantes.

- O número de comentários também não é proporcional à porcentagem do filme assistido. Os comentários dependem diretamente do interesse do participante em obter o entendimento da história.

- A descrição do fragmento depende diretamente do estado emocional do participante.

- Alguns participantes precisam de incentivos e lembretes para participar mais ativamente.

- A profissão dos participantes pode interferir na descrição das cenas.

- O TellStory não fornece ao facilitador da história nenhum feedback da participação dos membros.

- O TellStory não fornece um mecanismo de administração global das histórias, que gere relatórios, como por exemplo, número de linhas escritas e participação dos membros.

Outro ponto importante para a definição do mecanismo TellStory-Analyzer foi uma entrevista feita com os participantes na qual foi perguntado sobre possíveis melhorias na interação entre os usuários e de como motivá-los a participar mais ativamente das histórias. Nessas entrevistas foram constatados os seguintes pontos a serem melhorados no TellStory:

- Envio de e-mails a cada novo fragmento ou comentário inserido. 
- Possibilidade de uso de anonimato na descrição de um fragmento.

- Prêmios para as contribuições mais relevantes.

- Feedback sobre a participação dos usuários.

- Lembretes sobre o período em que o relato da história deve ser feito.

- Votação dos melhores fragmentos.

Baseado nessas análises, nas entrevistas e nos estudos dos dados obtidos, podese constatar que um mecanismo de administração das histórias que permita analisar a participação qualitativa e quantitativa do grupo seria de enorme valia para o facilitador da história. A partir disso, foi desenvolvido o mecanismo TellStory-Analyzer, com o objetivo de suprir algumas lacunas deixadas pelo TellStory e possibilitar uma maior externalização do conhecimento.

\section{TellStory-Analyzer}

O coordenador ou facilitador de uma história tem a função de [Borges e Pino, 1999] motivar idéias; manter o grupo motivado; promover a interação entre os participantes; proporcionar meios e formas para uma melhor comunicação; consolidar as idéias; manter a harmonia do grupo; e manter o controle de qualidade. Para apoiar estas funções, o mecanismo descrito neste artigo tem por objetivo fornecer um feedback para os membros do TellStory, principalmente aos facilitadores das histórias, permitindo um trabalho de gerência e motivação dos demais participantes. Além disso, esse mecanismo possui funcionalidades de gerenciamento e organização das histórias e dos membros, não implementados na ferramenta TellStory. A seguir serão descritas algumas funcionalidades desse mecanismo e também as suas contribuições.

O primeiro objetivo do mecanismo é fornecer aos usuários um feedback da participação. Para isso, esse mecanismo possui:

- Histórico de um determinado membro na ferramenta TellStory (Figura 1).

Nesta parte do mecanismo é possível vislumbrar em quais histórias um membro participa ou participou e o grau de atividade dele. Isso permite a escolha de um membro para contribuir em uma história através de sua experiência no assunto e também que o facilitador tenha controle sobre quais histórias estão sendo trabalhadas e quem são seus responsáveis.

- Estatísticas individuais sobre cada usuário de uma determinada história (Figura 2), podendo ser visualizado somente por facilitadores ou usuários comuns da história.

Os indicadores utilizados pelo mecanismo para a análise quantitativa e qualitativa são: número de fragmentos e comentários escritos e o total e a média de palavras escritas em cada fragmento ou comentário. A análise fornece um auxílio aos facilitadores para verificar como está sendo a contribuição e participação. A contribuição pode ser obtida pelo número de fragmentos e pelo total de texto escrito nos fragmentos. Já a participação pode ser calculada pelo número de comentários e volume de texto escrito nestes, pois para poder 
comentar o participante precisará ler os fragmentos escritos pelos demais participantes.

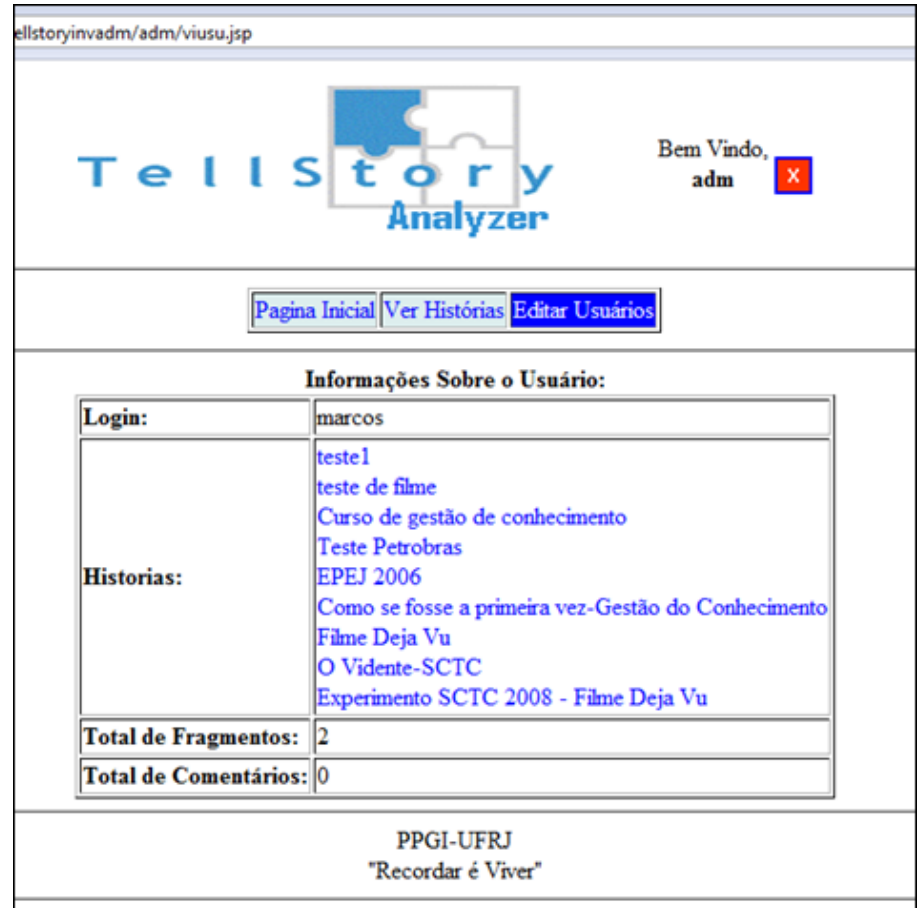

Figura 1. Histórico de participação de um usuário na ferramenta TellStory

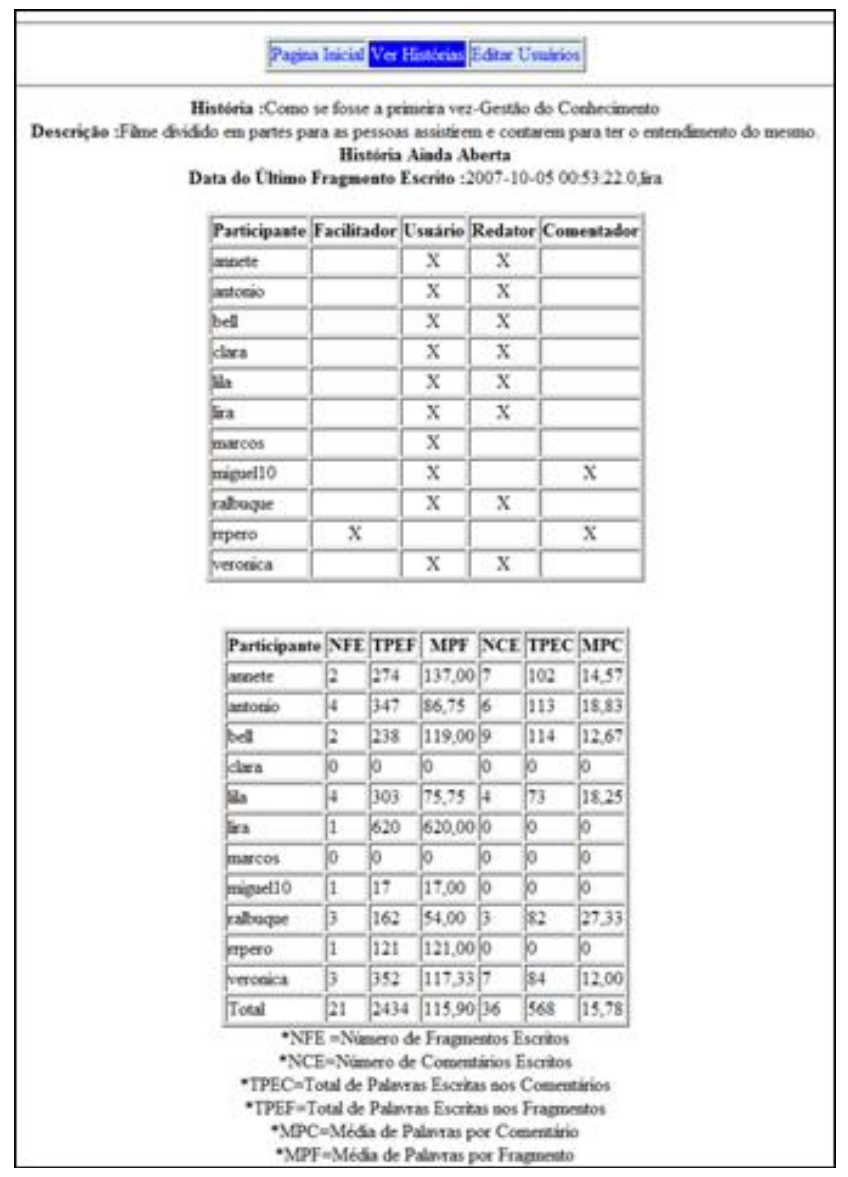

Figura 2. Estatísticas da História 
Cada uma das estatísticas individuais descritas anteriormente também pode ser exportada em formato de planilha para uma visualização gráfica, conforme mostrado na Figura 3. Os gráficos plotados nesta parte do mecanismo visam simplificar o trabalho do facilitador para o cálculo da participação e da contribuição dos membros da história. Alguns exemplos de gráficos criados a partir desse mecanismo são: gráficos sobre a porcentagem de fragmentos e comentários escritos por cada usuário. Isso contribui ainda mais para uma análise na linha do "participômetro" e do "contributômetro" dos membros da história, uma vez que a visualização de forma gráfica permite uma visão mais clara da participação e da contribuição de cada usuário.

Outro objetivo desse mecanismo é a gerência e organização do conteúdo do TellStory. Para isso, o TellStory-Analyzer possui estatísticas gerais sobre toda a base de dados do TellStory. Essas estatísticas mostram, por exemplo, a data e o autor do último fragmento e comentário escrito em cada uma das histórias (Figura 4). Além disso, o mecanismo também permite a gerência de todas as histórias e usuários, facilitando, assim, a criação eficaz de relatórios, recuperação de senhas, adição ou remoção de um usuário ou de uma história, atribuição de papéis nas histórias (facilitador, relator, usuário comum) e também inclusão ou exclusão de membros em uma determinada história.

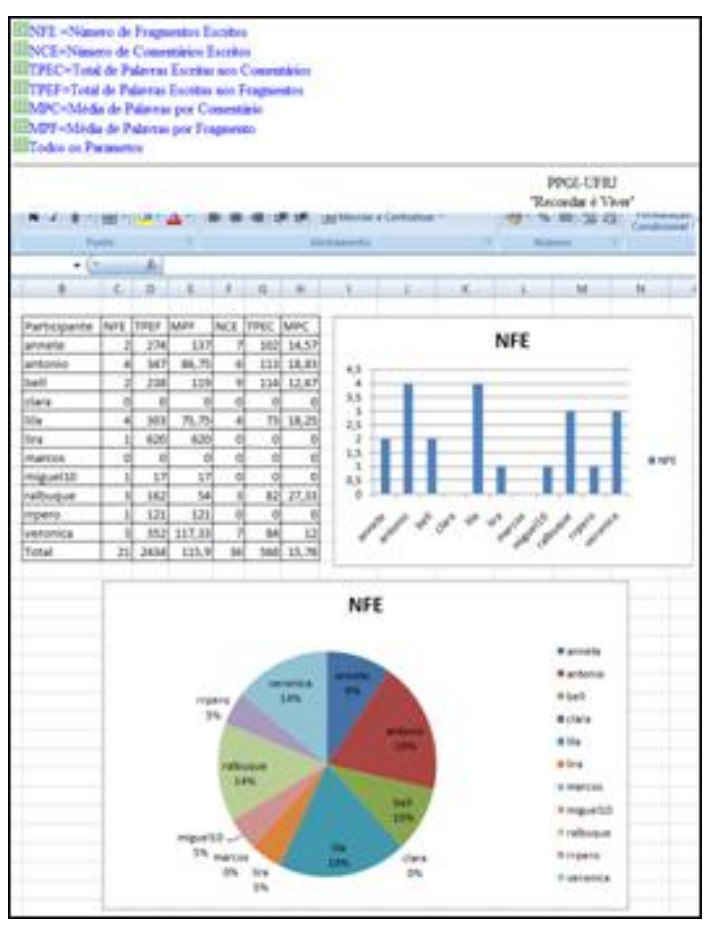

Figura 3. Visualização gráfica dos dados

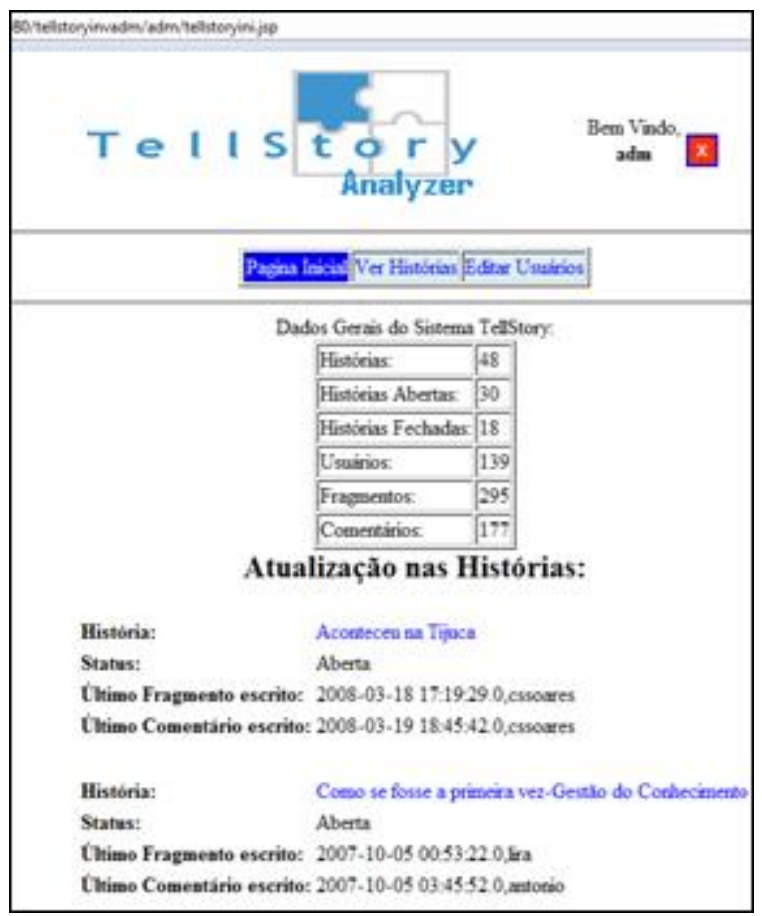

Figura 4. Estatísticas sobre a base de dados

Outro ponto relevante desse mecanismo é a recuperação de informação nas histórias, que permite aos membros procurar por trechos de frases ou por marcações (tags) de relevância cadastradas pelos facilitadores das histórias. Com isso, pode-se descobrir histórias similares e recuperar uma família de histórias sobre um mesmo tema. Por exemplo, caso o facilitador da história quisesse descobrir histórias que tratem de gestão de emergência, poderia fazer uma busca sobre esse tema (Figura 5). 
O uso desse mecanismo de recuperação de informação permite ao facilitador procurar e adicionar à história membros com experiência sobre um determinado assunto. A avaliação da participação de cada membro, para uma posterior busca, é feita pelo facilitador da história antiga através de uma pontuação atribuída à competência e à experiência de cada usuário naquela história. Além disso, ao término de uma história, o facilitador atribui tags à história (contendo as palavras chaves) para permitir uma maior precisão ao mecanismo de busca.

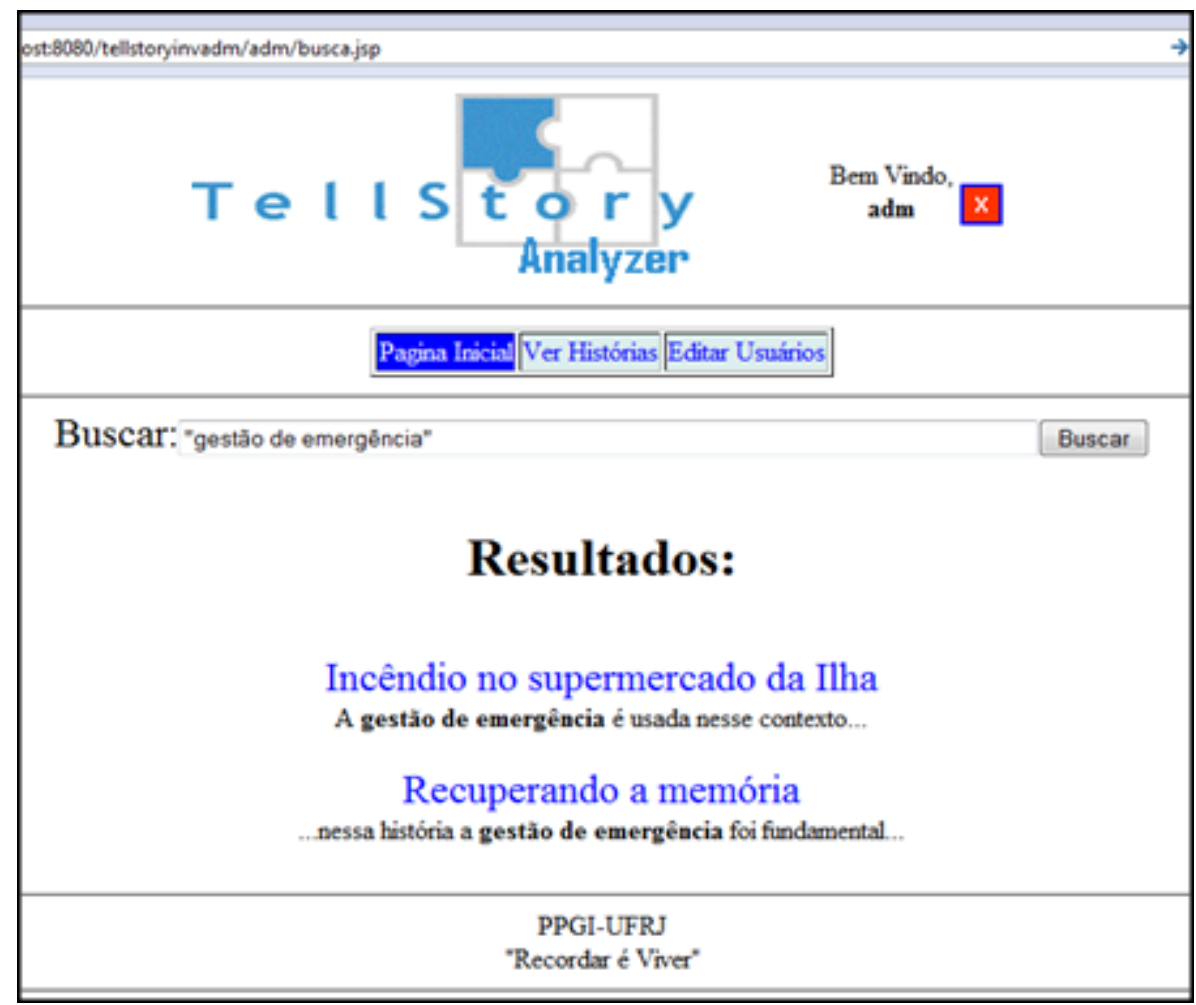

Figura 5. Recuperação de informação nas histórias

\section{Avaliação do TellStory-Analyzer}

Para avaliar o mecanismo TellStory-Analyzer e com o objetivo de demonstrar que o mecanismo de administração e análise quantitativa e qualitativa contribui para o enriquecimento das histórias, foi realizado um terceiro experimento. Assim como os outros dois experimentos, o terceiro experimento também foi realizado a partir da avaliação de um filme. Os integrantes do grupo assistiam a trechos desse filme com a intenção de reconstruí-lo no final.

$\mathrm{Na}$ realização desse experimento foram seguidas as etapas e os métodos propostos nos outros dois casos. Para participar dessa dinâmica, foram selecionados 8 participantes, sendo que 6 assistiram a trechos do filme e relataram os fragmentos, 1 teve a função de relator e 1 teve a função de monitorar a externalização do conhecimento através do mecanismo TellStory-Analyzer. O perfil dos participantes foi o seguinte: 2 professores (50 e 52 anos), 1 médico ( 28 anos), 1 secretária ( 27 anos), 2 
aposentados (62 e 78 anos), 1 estudante ( 25 anos, relator) e 1 analista de sistemas (24 anos, facilitador).

O mecanismo TellStory-Analyzer foi utilizado pelo facilitador, que não assistiu ao filme. Durante todo o período do experimento, o facilitador propôs questões sobre lacunas deixadas pelos outros membros, instigando-os a contribuírem mais. Além disso, ele estabeleceu uma ordem cronológica e uma classificação para os fragmentos a partir do conhecimento adquirido através da leitura dos fragmentos e dos questionamentos feito aos demais membros.

Após a realização do terceiro experimento, foi possível constatar um enriquecimento (tanto qualitativo quanto quantitativo) da história e da participação dos membros, conforme mostrado na Tabela 1. Nesse experimento, foi possível praticamente reconstruir a história total do filme. O critério usado para essa avaliação foi a escolha de duas pessoas que assistiram aos filmes inteiros e utilizaram como critério de avaliação a semelhança entre o filme e o relato escrito, atribuindo uma nota na escala de 0 a 10 à essa semelhança.

Ficou comprovado através da aplicação de um questionário, que o melhor desempenho do terceiro experimento foi devido à cobrança e questionamentos do facilitador, permitindo com isso uma maior interação, motivação e comprometimento do grupo. Vale ressaltar que quando questionados sobre o que mais os motivou a participarem, cinco dos seis participantes disseram que foi o interesse e também os questionamentos do facilitador, os quais os fizeram lembrar-se de coisas que haviam esquecido de relatar.

Tabela 1. Itens Avaliados nos Experimentos.

\begin{tabular}{|c|c|c|c|}
\hline Item Avaliado & $1^{\circ}$ Experimento & $2^{\circ}$ Experimento & $3^{\circ}$ Experimento \\
\hline Número de Fragmentos & 20 & 28 & 38 \\
\hline Número de Comentários & 36 & 25 & 68 \\
\hline Média de Palavras escritas por Fragmentos & 121,50 & 153,54 & 151,65 \\
\hline Média de Palavras escritas por Comentário & 17,78 & 23,88 & 33,90 \\
\hline Nota atribuída à história resgatada ${ }^{2}$ & 6,5 & 8,0 & 9,5 \\
\hline
\end{tabular}

\section{Conclusões}

A externalização do conhecimento é importante para a gestão do conhecimento. Esse é um dos temas amplamente tratados no grupo $\mathrm{GRECO}^{3}$ : gerenciar o conhecimento de uma maneira eficaz. Conhecimentos adquiridos anteriormente podem ser usados para uma série de finalidades, como, por exemplo, para tomada de decisões e para resolução de problemas futuros. O grupo GRECO vem utilizando o TellStory para recuperação de conhecimento.

Através dos dois primeiros experimentos apresentados neste artigo, foi possível identificar funcionalidades que pudessem auxiliar membros das histórias do TellStory a participarem mais ativamente e interagirem entre si, possibilitando com isso um ganho na externalização do conhecimento. A partir dessas funcionalidades, foi desenvolvido o mecanismo TellStory-Analyzer, cujo principal objetivo é fazer uma análise quantitativa e qualitativa das histórias, a fim de fornecer um feedback aos membros da história,

\footnotetext{
${ }^{2}$ Essa nota foi a média das notas atribuídas por duas pessoas que assistiram aos filmes inteiros e utilizaram como critério de avaliação a semelhança entre o filme e o relato escrito.

${ }^{3}$ www.greco.ppgi.ufrj.br
} 
principalmente ao facilitador. Esse mecanismo auxilia o trabalho do facilitador de motivação dos membros das histórias e também a agregação de conhecimento.

A eficiência do TellStory-Analyzer e a comprovação da hipótese de que o mecanismo de administração de história pode enriquecê-las pôde ser comprovada através do terceiro experimento que mostrou um ganho de qualidade e quantidade dos fragmentos e comentários escritos e também maior motivação e comprometimento dos membros do grupo. Além disso, com a utilização do TellStory-Analyzer pode-se constatar também um ganho efetivo no gerenciamento e organização da base de dados no TellStory.

É muito importante a criação de meios para estimular os indivíduos a participarem e interagirem com o grupo. O mecanismo desenvolvido durante este trabalho é uma forma para possibilitar o aprimoramento dessa externalização na ferramenta TellStory. Ainda há diversos estudos a serem feitos para se conseguir aprimorar ainda mais os mecanismos de auxílio à externalização do conhecimento em diversas áreas. A proposta do TellStory é que com pequenos fragmentos com pouco conhecimento, pode-se chegar próximo ao conhecimento global do que está sendo relatado. Esse trabalho é como um pequeno fragmento do TellStory que junto com os demais conhecimentos pode permitir grandes resultados na pesquisa por mecanismos realmente eficazes e eficientes para a externalização do conhecimento.

Como implementações futuras ao TellStory e ao TellStory-Analyzer, sugere-se:

- A construção de um módulo que forneça apoio ao relator da história.

- Estatísticas de quais usuários leram quais fragmentos, permitindo assim verificar conclusões sobre a história de uma maneira mais eficiente.

- Envio de e-mail avisando aos usuários quando algo for atualizado, evitando que eles tenham que entrar na ferramenta constantemente para verificar atualizações.

- Votação para fragmentos conflitantes.

\section{Referências}

Alavi, M. e Leidner, D.E. (1999) "Knowledge Management Systems: Issues, Challenges, and Benefits", Communications of the AIS, vol. 1, article 7, pp. 1-38.

Borges, M.R.S e Pino, J.A (1999) "Awareness Mechanisms for Coordination in Asynchronous CSCW", In: Workshop on Information Technologies and Systems (WITS), Charlotte, EUA, pp. 69-74.

Carminatti, N. A (2006) "Recuperação Coletiva do Conhecimento Aplicada a Situações de Emergência", Dissertação Mestrado em Informática - Programa de PósGraduação em Informática, Universidade Federal do Rio de Janeiro, Rio de Janeiro, $107 \mathrm{f}$.

Carminatti, N.A., Borges, M.R.S e Gomes, J.O. (2005) "Collective Knowledge Recall: Benefits and Drawbacks", In: International Workshop on Groupware, Recife. Lecture Notes in Computer Science. Berlin, Heidelberg: Springer-Verlag, 2005, vol. 3706, pp. 216-231. 
Costa, P., Araujo, E. , Borges, M.R.S (2009) "Uma Nova Abordagem para Desenhar Processos através da Contagem de Histórias", In: Workshop de Gestão de Processos de Negócio (WBPM), Fortaleza, Ceará.

Laporti, V., Borges, M. e Braganholo, V. (2009) "Athena: A collaborative approach to requirements elicitation”, Computers in Industry, vol. 60, pp. 367-380.

Nonaka, I. (1991) “The knowledge-creating Company”. Harvard Business Review, pp. 96-104.

Paula, D.R. e Cianconi, R.B. (2007) "Práticas de Gestão do Conhecimento: caso dos sítios associados ao portal corporativo da Fiocruz", Perspectivas em Ciência da Informação. vol. 12, n. 2, pp. 49-63.

Prusak, L. (2001) “Where Did Knowledge Management Come From?”, IBM Systems Journal, vol. 40, n. 4, pp. 1002-1007.

Perret, R. (2004) "A Técnica de Group Storytelling Aplicada à Gestão de Conhecimento", Dissertação Mestrado em Informática - Programa de PósGraduação em Informática, Universidade Federal do Rio de Janeiro, Rio de Janeiro, $189 \mathrm{f}$.

Perret, R., Borges, M. R. S. e Santoro, F. M. (2004) “Applying Group Storytelling in Knowledge Management", In: International Workshop on Groupware (CRIWG), San Carlos, Costa Rica. Groupware: Design, Implementation, and Use - Lecture Notes in Computer Science. Berlin: Springer-Verlag, vol. 3198. pp. 34-41.

Silva, S.A. (2006) "Modelação de Processos Alternativos de Conflito utilizando a Técnica de Storytelling", Dissertação de Mestrado em Engenharia Informática e de Computadores. Universidade Técnica de Lisboa Instituto Superior Técnico, Lisboa. Portugal.

Valle, C., Prinz, W. e Borges, M.R.S. (2002) "Generation of Group Storytelling in postdecision implementation process", In: International Conference on Computer Supported Cooperative Work in Design (CSCWD), Rio de Janeiro, pp. 361-367. 